\title{
Holography for automotive applications: from HUD to LIDAR
}

Pierre-Alexandre Blanche, Colton Bigler, Craig Draper, Joshua McDonald, Kalluri Sarma

Pierre-Alexandre Blanche, Colton Bigler, Craig Draper, Joshua McDonald, Kalluri Sarma, "Holography for automotive applications: from HUD to LIDAR," Proc. SPIE 10757, Optical Data Storage 2018: Industrial Optical Devices and Systems, 107570B (14 September 2018); doi: 10.1117/12.2323771

Event: SPIE Optical Engineering + Applications, 2018, San Diego, California, United States 


\title{
Holography for Automotive Applications: from HUD to LIDAR
}

\author{
Pierre-Alexandre Blanche ${ }^{a}$, Colton Bigler ${ }^{a}$, Craig Draper ${ }^{a}$, \\ Joshua McDonald $^{a}$, and Kalluri Sarma ${ }^{b}$ \\ ${ }^{a}$ College of Optical Sciences, The University of Arizona, \\ 1630 E University Blvd, Tucson, AZ 85721, USA. \\ ${ }^{b}$ Honeywell, Advanced Technology, 21111 N. 19th Avenue, \\ M/S 2J35, Phoenix, Arizona 85027, USA.
}

\begin{abstract}
Holography can offer unique solutions to the specific problems faced by automotive optical systems. Frequently, when possibilities have been exhausted using refractive and reflective designs, diffraction can come to the rescue by opening a new dimension to explore. Holographic optical elements (HOEs), for example, are thin film optics that can advantageously replace lenses, prisms, or mirrors. Head up display (HUD) and LIDAR for autonomous vehicles are two of the systems where our group have used HOEs to provide original answers to the limitations of classical optic. With HUD, HOEs address the problems of the limited field of view, and small eye box usually found in projection systems. Our approach is to recycle the light multiple times inside a waveguide so the combiner can be as large as the entire windshield. In this system, a hologram is used to inject a small image at one end of a waveguide, and another hologram is used to extract the image several times, providing an expanded eye box. In the case of LIDAR systems, non-mechanical beam scanning based on diffractive spatial light modulator (SLM), are only able to achieve an angular range of few degrees. We used multiplexed volume holograms (VH) to amplify the initial diffraction angle from the SLM to achieve up to $4 \pi$ steradian coverage in a compact form factor.
\end{abstract}

Keywords: Holography, holographic optical element, head up display, LIDAR, waveguide.

\section{INTRODUCTION}

Holographic optical elements (HOEs) are thin films of material where a diffraction pattern has been permanently recorded. This diffraction pattern can transform any incident wavefront to any predefined output wavefront. As such, HOEs can replace virtually any other optical element, such as lenses, prisms, or mirror. ${ }^{1,2}$ In addition to being very versatile, HOEs also offer the benefits of being thin, light, robust, and conformational. This later term describes the fact that HOEs can be coated onto non-planar surfaces, and conform to the shape of the support. These many advantages make HOEs extremely interesting in systems where the weight and size is limited, and where vibration can be an issue for other optical assembly. All these factors are extremely important in aerospace and automotive applications, which makes HOE a technology of choice for these environments.

The recording of HOE is achieved using an interferometric setup where a coherent laser beam is divided in two, forming a reference, and an object beam. ${ }^{3}$ The reference beam is usually uniform and collimated, while the wavefront of the object beam is shaped according to the wavefront that needs to be reproduced when the HOE is used. These two beams interfere inside the recording material, where the intensity interference pattern is imprinted as refractive index modulation. This index modulation is the diffraction pattern that will diffract the incident beam when the HOE is read.

There exist several holographic recording materials to manufacture HOES. ${ }^{4}$ The most commonly used nowadays is photopolymer such as the Covestro Bayfold ${ }^{\circledR}$, which is commercially available, benefits from a decent sensitivity $\left(10 \mathrm{~mJ} / \mathrm{cm}^{2}\right)$, and is easy to post process by simple UV (sun or lamp) exposure. Dichromated gelatin (DCG) is another material of choice that can achieve extremely high index modulation, and is robust to the

Send correspondence to P.-A.B.: pablanche@optics.arizona.edu

Optical Data Storage 2018: Industrial Optical Devices and Systems, edited by Ryuichi Katayama,

Yuzuru Takashima, Proc. of SPIE Vol. 10757, 107570B - @ 2018 SPIE

CCC code: $0277-786 \mathrm{X} / 18 / \$ 18 \cdot$ doi: $10.1117 / 12.2323771$

Proc. of SPIE Vol. 10757 107570B-1 
environment once encapsulated. ${ }^{5}$ However DCG has a very short shelf life that limits its distribution, has a low sensitivity $\left(100 \mathrm{~mJ} / \mathrm{cm}^{2}\right)$, and has a wet chemistry post-processing that is laborious to control.

The properties of HOEs, such as the efficiency $(\eta)$ and the angular $(\Delta \theta)$ or spectral $(\Delta \lambda)$ dispersion, depend on the materials physical parameters: thickness $(d)$, and index modulation $(\Delta n)$, as well as the diffraction pattern frequency $(\Lambda)$. The angular direction $(\theta)$ of the main diffraction order is given by the Bragg's law:

$$
\sin \theta=\frac{\lambda}{2 \Lambda}
$$

The calculation of the diffraction efficiency according to the incidence angle or the wavelength, is more tedious and requires the use of the rigorous coupled wave analysis. ${ }^{6}$ As an alternative, the Kogelnik coupled wave analysis theory, ${ }^{7}$ or the parallel stacked mirrors approximation developed by Brotherton-Ratcliffe ${ }^{8}$ both give very good results for a far simpler computation.

Some of the aberrations observed with HOEs due to media shrinkage/swelling, or due to the change of the light source between recording and reading can be calculated using the Bragg's law and some trigonometric relations between the point sources and image. ${ }^{1,9}$

In the following sections we present two specific examples where we have used HOEs to circumvent the limitations of the classical optical system that directly apply to automotive applications. The first one is a HUD with extended field of view and eye box. The second is a non-mechanical beam scanning system for LIDAR.

\section{HOLOGRAPHY FOR HUD}

Although HUDs provide major advantages in term of visibility of the information and driver awareness, ${ }^{10}$ the image displayed by HUD in automotive as well as aircraft has been kept relatively small due to the fact that, to increase its size, the relay optics that project the image onto the combiner need to become larger as well. It is simply not possible to house such large optics in either the dashboard of an automobile or the canopy of a plane.

\subsection{Holographic combiner}

Holographic combiners, using a reflective HOE, offer many benefits over other technologies. Their narrow bandwidth means they can let more outside light pass through, making them more transparent. They can diffract the light at a different angle than the Fresnel reflection from the substrate, avoiding the disturbing double image that can be observed with dichroic filters. As we have mentioned in the introduction, they can be conformational and applied directly to the curved windscreen or canopy. And lastly, they can act as the last collimation lens, which allows for a larger image than if that lens was in the projection system. These holographic combiners have been successfully used in both HUD and head mounted display (HMD). ${ }^{11-16}$

Figure 1 presents the recording and use of a conformational holographic combiner. To avoid aberrations, the object beam used in the recording setup should have the same wavefront as the reading beam. To provide accommodation at infinity, the reference beam should be collimated. The diffracted beam reproduces the same wavefront (complex conjugate) as the initial reference beam.

Since some people wonder about how to update the holographic image displayed by the combiner, it should be pointed out that the holographic combiner is a reflective hologram that does not present any image per se. Rather, the combiner acts as a mirror where the image from the projector is reflected onto.

\subsection{Waveguide system}

More recently, waveguide optics have been used to improve upon the traditional reflective holographic combiner. ${ }^{17-20}$ The unique property of this system is that the light that travels inside the waveguide using the total internal reflection (TIR) condition can be recirculated and extracted several times. This mechanism increases the size of the pupil to offer a larger eye box. A schematic representation of this process is presented in figure 2 .

Bears noting that there exist several conditions for the successful expansion of the pupil using a waveguide: for the technique to present one single image, instead of multiple copies of the same image, the extraction should be collimated, which puts the image at infinity. Luckily, this is the type of configuration mostly used for 

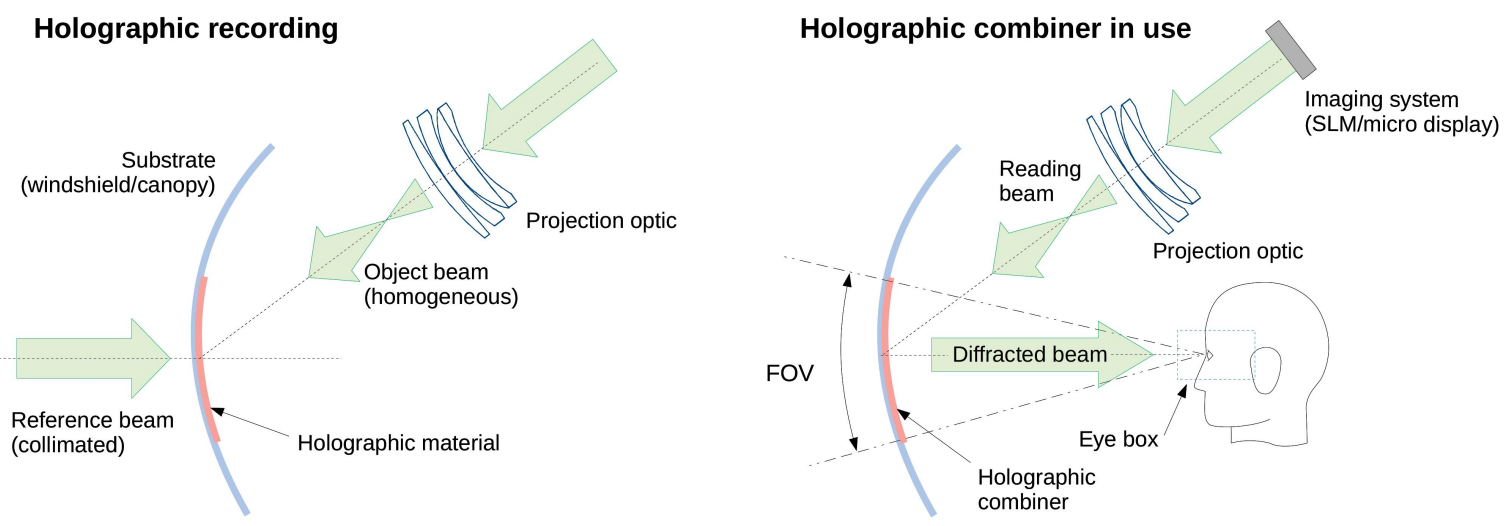

Figure 1. Recording (left) and use (right) of a conformational holographic combiner.

Imaging device

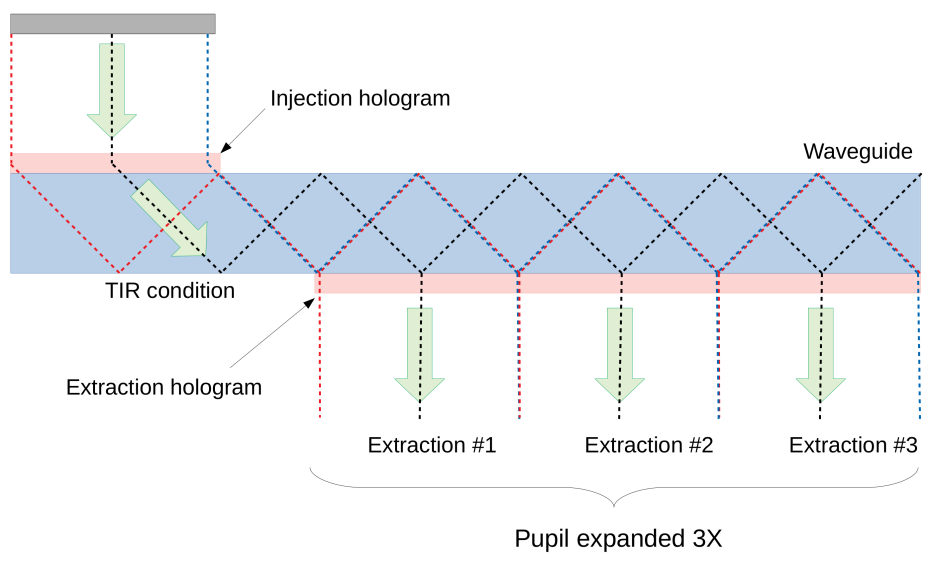

Figure 2. Use of a waveguide for pupil expansion.

HUD. The image propagating inside the waveguide also needs to be collimated. If not, the different extractions would present an image with different magnifications due to the continuous expansion of the image during its propagation inside the waveguide. To make sure there is no gap between the different extractions, the left-most part of the image (red beam in fig. 2) should reflect back to the right-most part of the image (blue beam in fig. 2). This condition, which is also linked to the TIR critical angle, connects together the minimum size of the image at the injection hologram $\left(L_{m} i n\right)$ with the thickness $(d)$ and index of refraction $(n)$ of the waveguide:

$$
L_{m} i n=2 d \tan [\arcsin (1 / n)]
$$

Finally, to balance the intensity of the different extractions, the efficiency of the extraction hologram should increase along the length of the waveguide. This is because the amount of light inside the waveguide $(I(n))$ decreases after each partial extraction of the image as:

$$
I(n)=I_{0}\left(1-\frac{n-1}{N}\right)
$$

With $I_{0}$ is the initial intensity, $N$ the number of extractions, and $n$ the extraction number. be:

For each extraction to have an equal intensity of $I / N$ the diffraction efficiency $\eta$ of the $n^{\text {th }}$ hologram should

$$
\eta(n)=\frac{1}{N-n+1}
$$


Equations 3 and 4 are presented in figure 3.

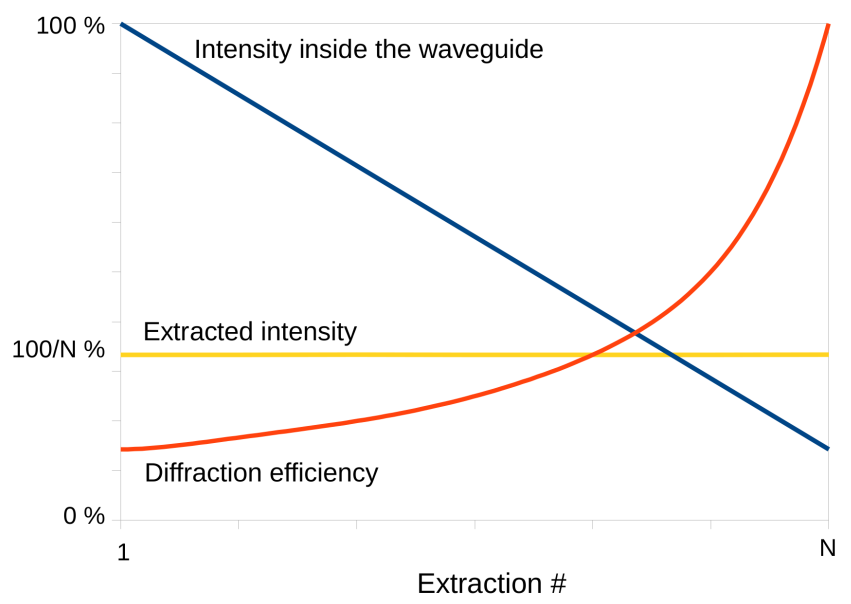

Figure 3. Evolution the diffraction efficiency of the extraction hologram to compensate for the reduction of intensity inside the waveguide, and keep the extracted intensity constant.

It is possible to generalize the principle of the pupil expansion in two dimensions by adding a redirection hologram in the waveguide architecture. This configuration is presented in figure 4

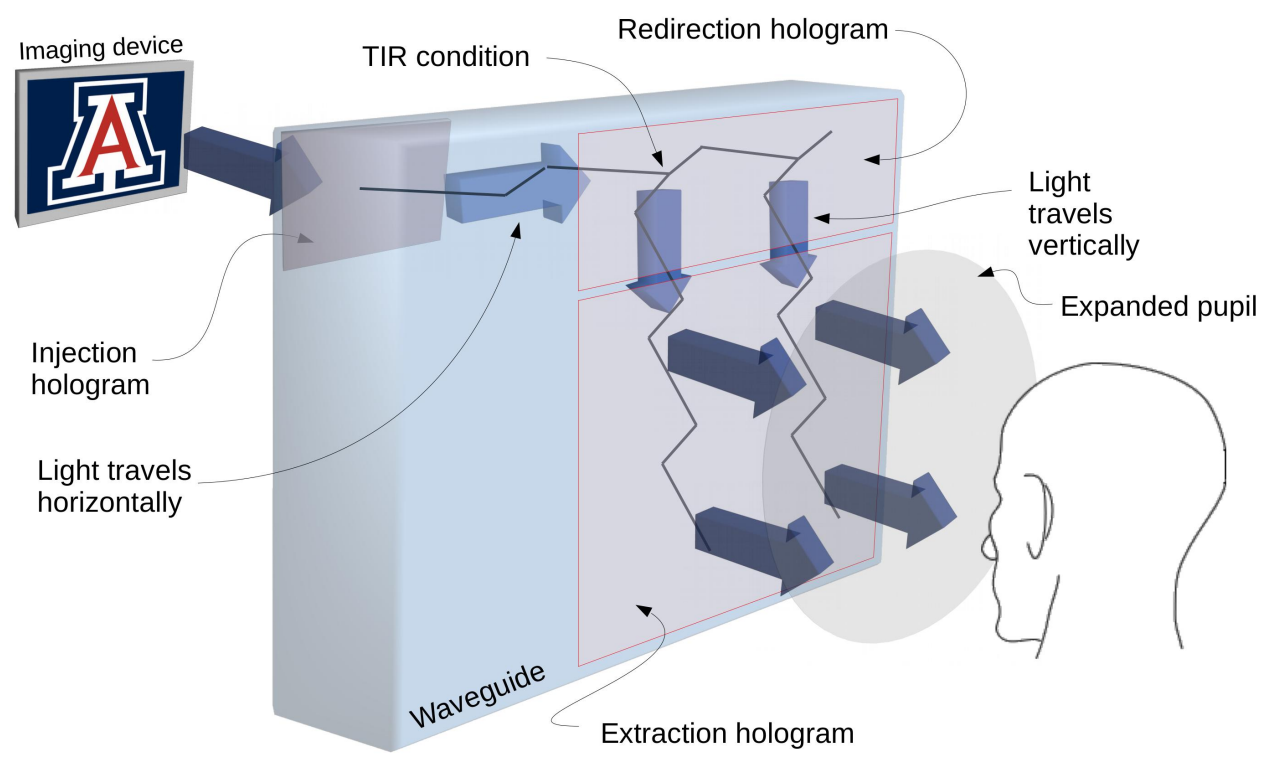

Figure 4. Two dimensional pupil expansion using waveguide propagation and 3 holograms: injection, redirection, and extraction.

\section{HOLOGRAPHY FOR LIDAR}

With the introduction of autonomous vehicles, and the explosion of drones for civilian applications, light detection and ranging (LIDAR) uses for collision avoidance and topological measurement is becoming ubiquitous. ${ }^{21-24}$ 


\subsection{Non-mechanical beam steering}

The dominant beam steering mechanism for LIDAR scanners is a galvanometric mirror system. However, nonmechanical beam steering (NMBS) technologies are a particularly interesting alternative, since they are not prone to mechanical failure due to fatigue; they are faster, lighter, and can generate arbitrary scanning patterns including multicasting (multiple beams). They are also more compact which, in the case of automotive vehicles, can be highly appreciated. We all have seen these pictures of early self-driving cars with a spinning mirror on the roof, and no one wants their next vehicle looking like that.

Existing technologies for NMBS include liquid crystal on silicon (LCoS), micro electro-mechanical systems (MEMS), phase delay array (PDA), electro-optic modulator (EOM), and acousto-optics modulators (AOM). ${ }^{25,26}$ Although these technologies are all very promising, they all have a common weakness that impedes their adoption: their maximum sweeping angle is severely limited. This sweeping angle varies with the technology: from a few degrees (LCoS, MEMS, PDA), to around ten degrees (EOM, AOM), but certainly does not cover the large field of view required for automotive LIDAR applications.

The root cause of the limited steering angle for NMBS systems can be understood by examining the fundamental physics of scanning system. In these devices, the steering is accomplished by introducing a spatial phase modulation in the transverse section of the beam. According to the frequency $(\Lambda)$ of this modulation, the beam is diffracted to a specific angle $(\theta)$ that can be calculated using the Bragg law given in equation 1.

The maximum diffraction angle that can be achieved by an SLM/PDA occurs when displaying a binary grating. Using $4 \mu \mathrm{m}$ as a typical pixel pitch value for the SLM/PDA, the binary grating has an $8 \mu \mathrm{m}$ periodicity, or $125 \mathrm{lp} / \mathrm{mm}$ frequency. For a $1550 \mathrm{~nm}$ wavelength (c-band telecommunication) the maximum diffraction angle found using eq. 1 is $\pm 5.5^{\circ}$. When integrated in both directions, this corresponds to 3E-2 steradian. EOMs and AOMs are able to deliver larger angles, but are still limited to approximately $\pm 10^{\circ}$, or $0.1 \mathrm{sr}$. For the commercial applications such as LIDAR, a scanning angle of at least $\pi$ steradian is required.

The scanning range becomes even smaller when using a blazed grating. A blazed grating has a saw-tooth profile and requires more pixels, or emitters, to form the diffraction pattern on the SLM/PDA. The use of blaze gratings is strongly desired for beam steering because their efficiency is much higher than binary grating. ${ }^{27}$ The equation governing the diffraction efficiency $(\eta)$ according to the number of discrete levels $(N)$ used to reproduce the sawtooth function is:

$$
\eta=\operatorname{sinc}^{2}(1 / N)
$$

It can be seen in figure 5 where equations 1 and 5 are plotted simultaneously, that when the number of pixels defining the grating increases, the efficiency increases, but the diffraction angle decreases.

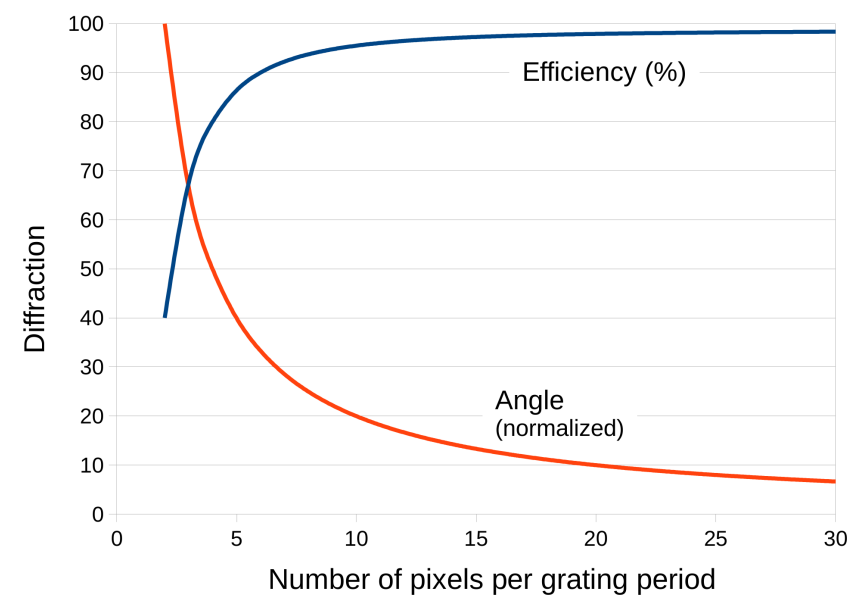

Figure 5. Diffraction efficiency and diffraction angle according to the number of pixels used to define the blazed grating frequency. 
The fundamental reason why non-mechanical beam steering devices are limited to a few degrees of angular amplitude derives from the pixel pitch and the Braggs law, and cannot be overcome by simple improvements in the underlying technologies.

\subsection{Holographic amplification of the scanning angle}

A complementary system that amplifies the diffracted angle to cover a significant portion of the sphere (solid angle) is required to bring the benefits of NMBS system to fruition.

One possible approach to amplify the NMBS small scanning angle would be to place a simple refractive lens in the output optical path. However, in this approach, the beam would not be collimated but diverging, which prevents its use in LIDAR applications. To keep the beam collimated, a second lens is needed to form a telescope. Unfortunately, this configuration is bulky and restricts the field of view to unacceptable levels due to the conservation of étendue.

Holography can come to the rescue once again by using multiplexed Bragg gratings. Bragg gratings are volume holograms that are highly selective according to the angle of incidence of the light: only the light arriving at a particular angle is diffracted to the desired direction by the grating. The light that is incident to other angles passes through the grating unaffected. By superimposing several Bragg gratings, or alternatively, by multiplexing several Bragg gratings into the same material, it is possible to redirect the light incident at different discrete angles to other arbitrary angles. The diffraction angles can be much larger than the incident angles, making it possible to redirect the light in predetermined direction. A schematic of such a system with two Bragg gratings is present in figure 6 .

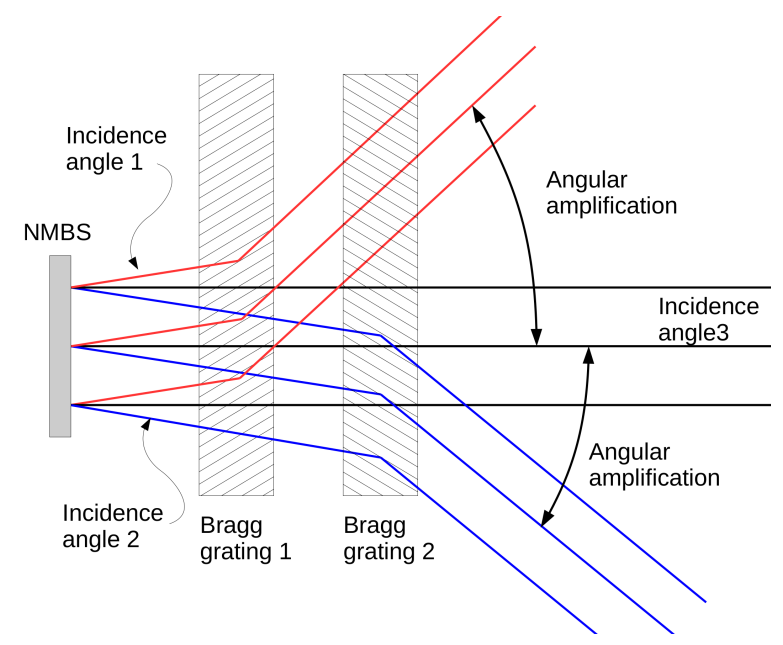

Figure 6. Schematic of 2 Bragg gratings used to diffract the light incident at different angles to different directions. The gratings can be multiplexed inside the same hologram.

The number of incident angles that the system can redirect is equal to the number of Bragg gratings that are present. Other angles are transmitted through the system and the light is not lost. The angular selectivity of a Bragg grating can be computed using the Kogelnik theory of coupled wave analysis. ${ }^{7}$ In first approximation, the angular selectivity is inversely proportional to the thickness of the grating. Figure 7 shows the diffraction efficiency according to the angle of incidence for Bragg gratings with different thicknesses $(d)$ in microns. The Bragg angle i.e. the angle with maximum efficiency is kept constant at $5^{\circ}$. To accommodate for the thickness increase, the index modulation $(\Delta n)$ is reduced proportionally.

Coupled wave theory computation, presented in figure 8 , shows that for an angular selectivity of $0.1^{\circ}$, which is achievable with NMBS devices, an amplification factor of 10 for the output angle, and a cross talk $<8 \mathrm{~dB}$ between the incident angles, which is required for LIDAR application, the thickness of the Bragg grating should be $10 \mathrm{~mm}$. This computation assumes a wavelength of $1550 \mathrm{~nm}$, which is the preferred band of operation for 


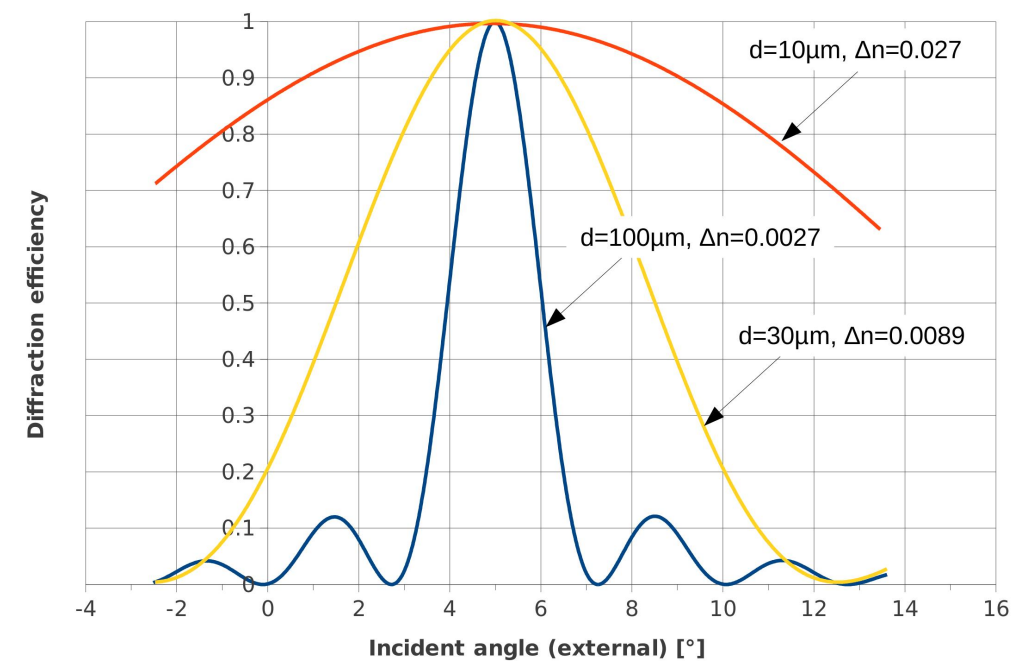

Figure 7. Computation of the diffraction efficiency according to the angle of incidence for Bragg gratings with different thicknesses: $10 \mu \mathrm{m}, 30 \mu \mathrm{m}, 100 \mu \mathrm{m}$. FWHM reduced when thickness increases.

LIDAR (eye safety). Better isolation or a larger number of angles can be achieved with thicker holograms, or by stacking multiple holograms on top of each other.

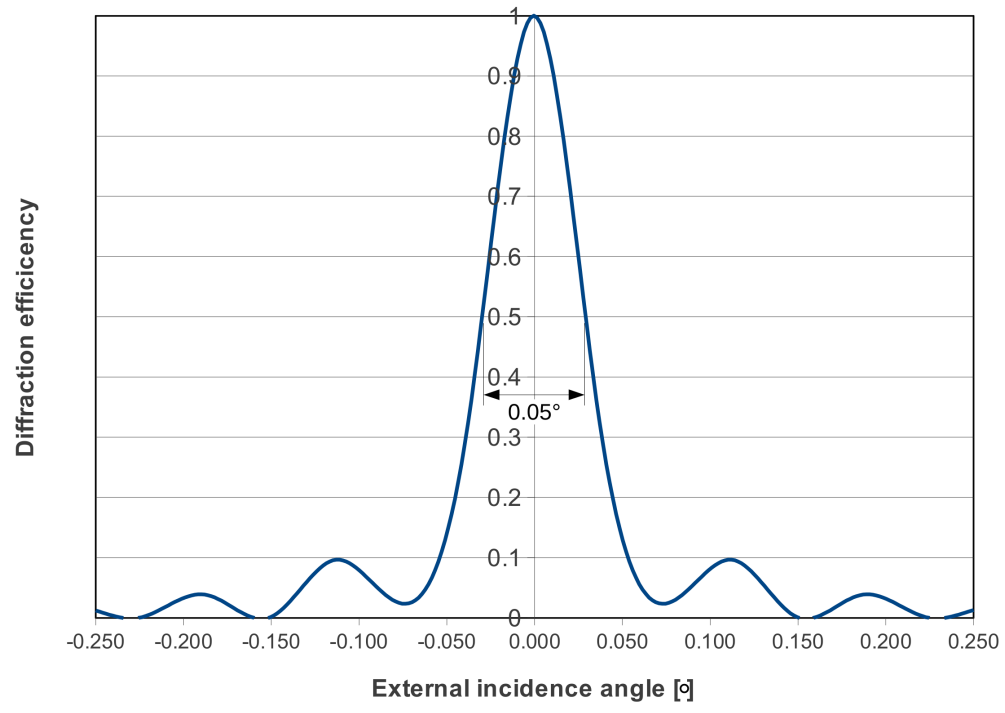

Figure 8. Angular selectivity for a $10 \mathrm{~mm}$ thick Bragg grating.

By itself, the layer of multiplexed Bragg gratings can expand the angular coverage of the scanning system up $2 \pi$ steradian, which is already significant. It is furthermore possible to double that coverage up to the entire sphere: $4 \pi$ steradian, by the addition of a hemispherical lens on the top of the Bragg gratings. This system is presented in figure 9 where another layer of holographic gratings is coated on top of the hemisphere to redirect and collimate the beams. 


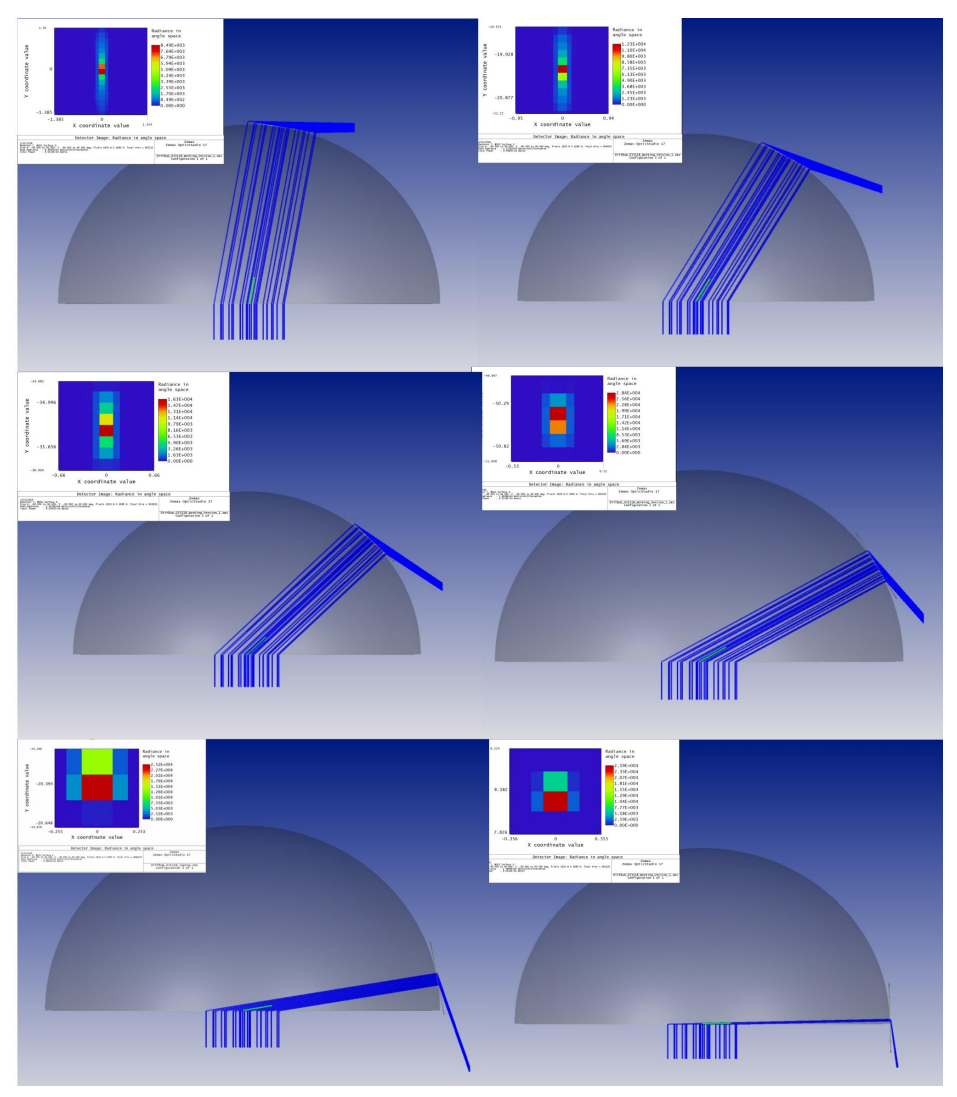

Figure 9. Ray tracing model showing the amplification of the scanning angle up to retro-reflection (bottom right). Insets are the associated angular radiance profile of the beams, showing beam collimation.

\section{CONCLUSION}

In the framework of automotive applications, where there exist rigorous constrains of space, weight, and robustness, holographic optical elements can provide unique solutions to otherwise intractable problems.

For HUD, holography allows large and conformational combiner to be coated directly on the windshield. These diffractive elements operate outside the laws of simple reflection, which allows for image correction and eliminates the double reflection produced by other techniques. Using waveguide optics, the combiner can also be used to expand the size of the pupil. In this case, the holograms act as injection, redirection, and extraction elements, which further reduces the size of the overall system.

For LIDAR scanners, used in autonomous vehicle, holography can be used to make fast, robust, and compact non-mechanical beam steering device. However, so-far, these promising devices only offer steering angles of a few degrees. We propose to use multiplexed Bragg gratings on top of the steering device to amplify this angle. We demonstrated, using ray tracing, that such a system can cover up to $4 \pi$ steradian. Our next step will be to make a demonstrator for the technology.

\section{ACKNOWLEDGMENTS}

Authors acknowledge the support of the NSF under grants PFI:AIR-TT \#1640329, and ERC CIAN\#EEC0812072, Honeywell Labs under grants \#6400282571, and \#6400327460, as well as the Arizona Technology Research Initiative Fund (TRIF) Industry and WEES Link under grants \#5832500 and \#5835212 respectively. 


\section{REFERENCES}

[1] Blanche, P.-A., [Field Guide to Holography], SPIE press book (2014).

[2] Kim, N., Piao, Y.-L., and Wu, H.-Y., "Holographic optical elements and application," in [Holographic Materials and Optical Systems], Naydenova, I., ed., ch. 5, InTech, Rijeka (2017).

[3] Blanche, P.-A., [Holography], 259 - 297, CRC Press, second edition ed. (2017).

[4] Blanche, P.-A., "Holographic recording media and devices," in [Encyclopedia of Modern Optics (Second Edition)], Guenther, B. D. and Steel, D. G., eds., 87 - 101, Elsevier, Oxford, second edition ed. (2018).

[5] Blanche, P.-A., Habraken, S., Lemaire, P., and Jamar, C., "Diffracted wavefront measurement of a volume phase holographic grating at cryogenic temperature.," Applied optics 45(27), 6910-6913 (2006).

[6] Moharam, M. G. and Gaylord, T. K., "Three-dimensional vector coupled-wave analysis of planar-grating diffraction," Journal of the Optical Society of America 73(9), 1105 (1983).

[7] Kogelnik, H., "Coupled wave theory for thick hologram gratings," Bell System Technical Journal 48(9), 2909-2947 (1969).

[8] Brotherton-Ratcliffe, D., "A treatment of the general volume holographic grating as an array of parallel stacked mirrors," Journal of Modern Optics 59(13), 1113-1132 (2012).

[9] Benton, S. A. and Bove, V. M., [Holographic Imaging], Wiley (2008).

[10] Liu, Y.-C. and Wen, M.-H., "Comparison of head-up display (hud) vs. head-down display (hdd): driving performance of commercial vehicle operators in taiwan," International Journal of Human-Computer Studies 61(5), 679 - 697 (2004).

[11] Kress, B. and Shin, M., "Diffractive and holographic optics as optical combiners in head mounted displays," in [Proceedings of the 2013 ACM conference on Pervasive and ubiquitous computing adjunct publication], 1479-1482, ACM (2013).

[12] Kress, B. and Starner, T., "A review of head-mounted displays (hmd) technologies and applications for consumer electronics," in [Proc. SPIE], 8720, 87200A (2013).

[13] Guillaumee, M., Vahdati, S. P., Tremblay, E., Mader, A., Bernasconi, G., Cadarso, V. J., Grossenbacher, J., Brugger, J., Sprague, R., and Moser, C., "Curved holographic combiner for color head worn display," Journal of Display Technology 10(6), 444-449 (2014).

[14] Moon, E., Kim, M., Roh, J., Kim, H., and Hahn, J., "Holographic head-mounted display with rgb light emitting diode light source," Optics express 22(6), 6526-6534 (2014).

[15] Peng, H., Cheng, D., Han, J., Xu, C., Song, W., Ha, L., Yang, J., Hu, Q., and Wang, Y., "Design and fabrication of a holographic head-up display with asymmetric field of view," Appl. Opt. 53, H177-H185 (Oct 2014).

[16] Wood, R. B. and Howells, P. J., [Head-up Display], ch. 7, CRC Press, third edition ed. (2017).

[17] Cameron, A., "The application of holographic optical waveguide technology to the q-sight family of helmetmounted displays," in [Head-and Helmet-Mounted Displays XIV: Design and Applications], 7326, 73260H, International Society for Optics and Photonics (2009).

[18] Mukawa, H., Akutsu, K., Matsumura, I., Nakano, S., Yoshida, T., Kuwahara, M., and Aiki, K., "A fullcolor eyewear display using planar waveguides with reflection volume holograms," Journal of the society for information display 17(3), 185-193 (2009).

[19] Homan, M., "The use of optical waveguides in head up display (hud) applications," in [Display Technologies and Applications for Defense, Security, and Avionics VII], 8736, 87360E, International Society for Optics and Photonics (2013).

[20] Bigler, C. M., Blanche, P.-A., and Sarma, K., "Holographic waveguide heads-up display for longitudinal image magnification and pupil expansion," Applied optics 57(9), 2007-2013 (2018).

[21] Fleming, W. J., "New automotive sensorsa review," IEEE Sensors Journal 8(11), 1900-1921 (2008).

[22] Ilas, C., "Electronic sensing technologies for autonomous ground vehicles: A review," in [Advanced Topics in Electrical Engineering (ATEE), 2013 8th International Symposium on], 1-6, IEEE (2013).

[23] Ziebinski, A., Cupek, R., Grzechca, D., and Chruszczyk, L., "Review of advanced driver assistance systems (adas)," in [AIP Conference Proceedings], 1906(1), 120002, AIP Publishing (2017).

[24] Tang, L. and Shao, G., "Drone remote sensing for forestry research and practices," Journal of Forestry Research 26(4), 791-797 (2015). 
[25] McManamon, P. F., Bos, P. J., Escuti, M. J., Heikenfeld, J., Serati, S., Xie, H., and Watson, E. A., "A review of phased array steering for narrow-band electrooptical systems," Proceedings of the IEEE 97(6), 1078-1096 (2009).

[26] Keeler, G. A., "Nanoscale optical modulators: Application drivers and recent developments," in [2018 Optical Fiber Communications Conference and Exposition (OFC)], 1-3, IEEE (2018).

[27] Blanche, P.-A., LaComb, L., Wang, Y., and Wu, M. C., "Diffraction-based optical switching with mems," Applied Sciences 7(4), 411 (2017). 